\title{
Anomalous Biceps Femoris Tendon Insertion Leading to a Snapping Knee in a Young Male
}

\author{
Brett A Fritsch, FRACS (Orth) ${ }^{1}$ and Vikram Mhaskar, MS (Orth), MCh (Orth) ${ }^{2}$ \\ ${ }^{1}$ Sydney Orthopaedic Research Institute, Chatswood, Australia; ${ }^{2}$ Department of Knee \& Shoulder Surgery, Knee \& Shoulder Clinic, New Delhi, India
}

Snapping biceps femoris tendon is an uncommon problem that can be caused by various anatomical aberrations around the knee joint. There are several case reports in the literature describing some of these anatomical variations and their treatment. We present a case of unilateral snapping biceps femoris tendon due to a previously unreported anatomical variation, our technique for successful surgical treatment, and a review of the literature.

Keywords: Knee, Biceps femoris, Anomalous insertion, Snapping

Snapping biceps femoris tendon, though being a rare phenomenon, is not alien to clinical literature ${ }^{1-9)}$. The reported causes for this manifestation are varied, ranging from anomalous tendon anatomy ${ }^{1-9)}$ to trauma ${ }^{7}$. The major symptom is a painful click on the lateral side of the knee, generally during flexion. The initial treatment is usually conservative but surgical treatment has been shown to be successful in relieving symptoms when a conservative approach fails. Snapping affecting activities of daily living like sitting, ability to perform work, or playing a sport for an athlete or worsening after a trial of physical therapy, use of analgesics, and avoiding precipitating movements are indications for surgery. The surgical techniques described in the literature centre around recreating normal biceps insertion anatomy. In this paper, we describe a case of anomalous insertion of the biceps femoris tendon and a simple way of treating it operatively.

The anatomy of the biceps femoris insertion is complex. The

Received October 22, 2015; Revised (1st) November 18, 2015;

(2nd) June 1, 2016; Accepted June 15, 2016

Correspondence to: Vikram Mhaskar, MS (Orth), MCh (Orth)

Department of Knee \& Shoulder Surgery, Knee \& Shoulder Clinic, F7,

East of Kailash, New Delhi 110065, India

Tel: +91-011-2628-6869, Fax: +91-98-1880-6857

E-mail:drvikrammhaskar@gmail.com

This is an Open Access article distributed under the terms of the Creative Commons Attribution Non-Commercial License (http://creativecommons.org/licenses/by-nc/4.0/) which permits unrestricted non-commercial use, distribution, and reproduction in any medium, provided the original work is properly cited. most detailed dissection, described by Terry and LaPrade ${ }^{10)}$, identified no less than 11 discrete bands of insertion of the long and short heads. These are divided into tendinous and fascial components which insert to the fibular head, the posterolateral capsule, the lateral collateral ligament (LCL) itself, the iliotibial band (ITB), the fascia of the anterolateral compartment of the leg, and the anterolateral tibia up to Gerdy's tubercle. These multiple insertions are arranged in such a way as to envelope the distal 1/4 of the LCL and provide a complex adjunct to stability of the knee throughout the range of motion (ROM) without causing mechanical irritation as they are stretched and contracted during activity. Variations in the normal arrangement can result in pathological snapping and the key to its successful treatment lies in determining exactly where that variation has occurred and correcting it.

Here, we present a case of such a variation, previously undescribed, along with successful management. A systematic review of the literature encompassing other published variations and treatment methods is also included in order to aid management of future cases.

\section{Case Report}

An 18-year-old male patient presented with a painful snap over the lateral aspect of his knee whenever he flexed his knee and low grade ache over the lateral side of the knee following sitting 
down/squatting. He worked as a manual labourer and was a club level basketball player. He could consistently reproduce a clearly audible snap by contracting his biceps femoris muscle with the knee in between $80^{\circ}$ and $100^{\circ}$ of flexion. The symptoms had started spontaneously around 12 months back without any specific trauma, and had been getting worse over the past 6 months. It was preventing him from playing a sport and doing his job that involved squatting. He was initially treated conservatively by avoidance of activities that precipitate the symptoms; however, he was not responsive to physical therapy for 6 months and then symptoms got worse. He was not able to play basketball for 6 months, and had not been working for the last 3 months. His knee examination was normal except for a palpable movement of the biceps femoris tendon over the fibular head when the knee was taken through $80^{\circ}-120^{\circ}$ of flexion. The biceps tendon could be easily palpated and passively moved anteriorly and posteriorly over the fibular head, reproducing mechanical irritation in the distal tendon. Radiography and magnetic resonance imaging scans did not demonstrate any abnormality.

\section{Operative Findings and Technique}

The patient was given a general anaesthetic and was positioned supine with the knee at $60^{\circ}$ of flexion against a foot and thigh bolster. A $5 \mathrm{~cm}$ incision was made in line with the biceps tendon over the fibular head, ending at Gerdy's tubercle. Superficial and deep dissection exposed the common peroneal nerve at the level of the neck of the fibula, and a neurolysis was performed from the fibula neck back under the biceps tendon and into the posterior compartment of the thigh. The terminal insertions of the bi- ceps tendon were then carefully exposed and examined. In Terry and LaPrade ${ }^{10)}$ descriptions, the main tendon of the long head divides just proximal to the fibular head and gives an insertion to the posterolateral edge of the fibular head just posterior to the LCL insertion (the "direct arm" of the long head) before continuing on as a thinner anterior arm that ends by blending with aponeurosis of the anterior compartment of the leg as well as giving some deep fibres of its own to the lateral edge of the fibula, thus making a sling with the direct arm encompassing the LCL insertion (Fig. 1). In our case, it was the direct arm that was thin and relatively deficient, and the anterior arm was hypertrophic, cordlike, and inserted into the anterolateral fascia on the tibia that blended with the Gerdy's tubercle over the ITB insertion (Figs. 2 and 3). It was this thickened cord that moved from superior to the fibular head to inferior to the fibula as the knee was flexed, causing a loud "snap" as it flicked over the prominence of the fibular head at that ROM. There was also a hypertrophic lateral prominence of the fibular head.

The insertion of this hypertrophic anterior arm was carefully detached (Fig. 4) from the tibia and a No. 5 Ethibond (Ethicon, Somerville, NJ, USA) suture was whip stitched on its distal 20 $\mathrm{mm}$. The whipstitching involved taking five throws on either side of the detached tendon with $10 \mathrm{~cm}$ of two free suture ends at the distal most aspect of the tendon from where it was detached much like whipstitching hamstring tendons in an anterior cruciate ligament reconstruction. The prominent posterior-lateral border of the fibular styloid was identified and removed using a small osteotome (Fig. 5) and a rongeur was used to obtain a smooth edge. Using a guide wire and a $7 \mathrm{~mm}$ reamer, a socket

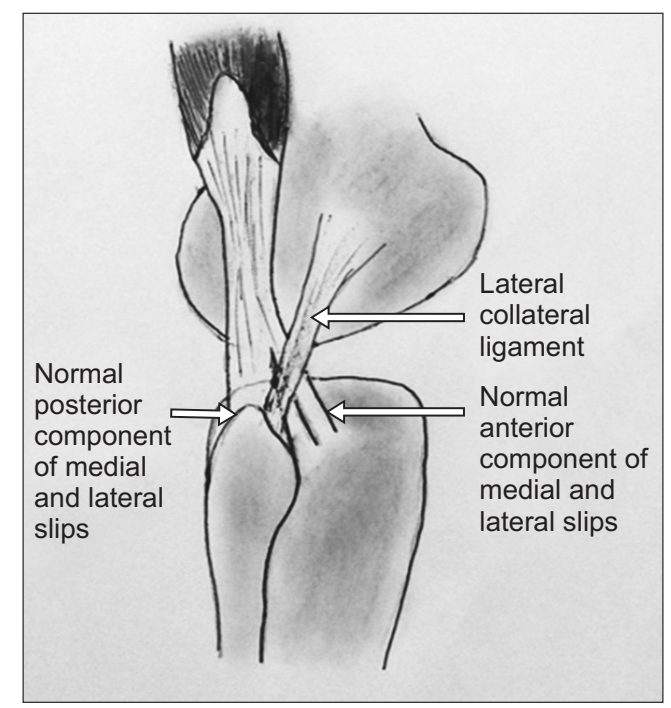

Fig. 1. Normal Biceps Femoris insertion.

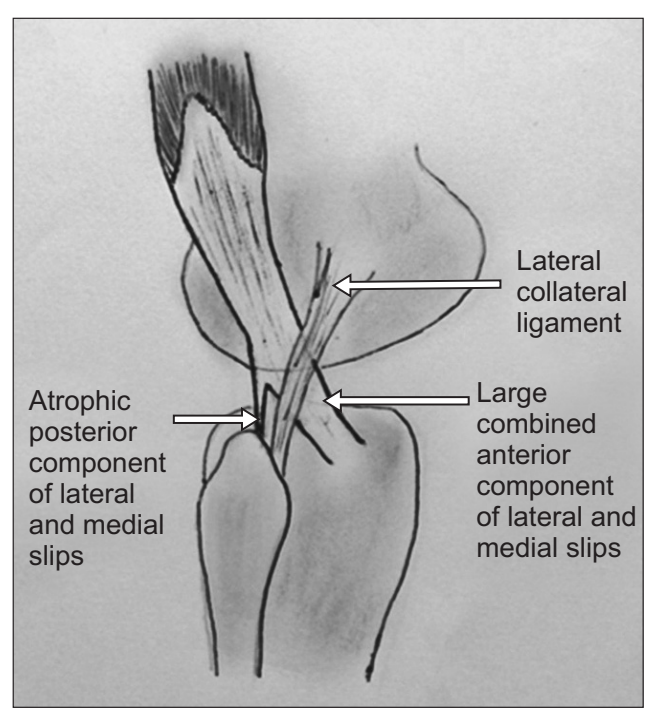

Fig. 2. Anomalous Biceps Femoris tendon insertion. 


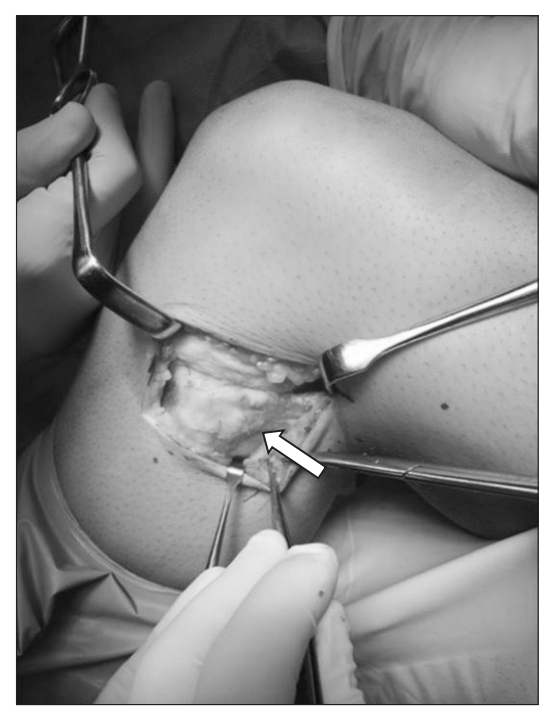

Fig. 3. Intraoperative picture of hypertrophied reflected arm of the biceps femoris tendon.

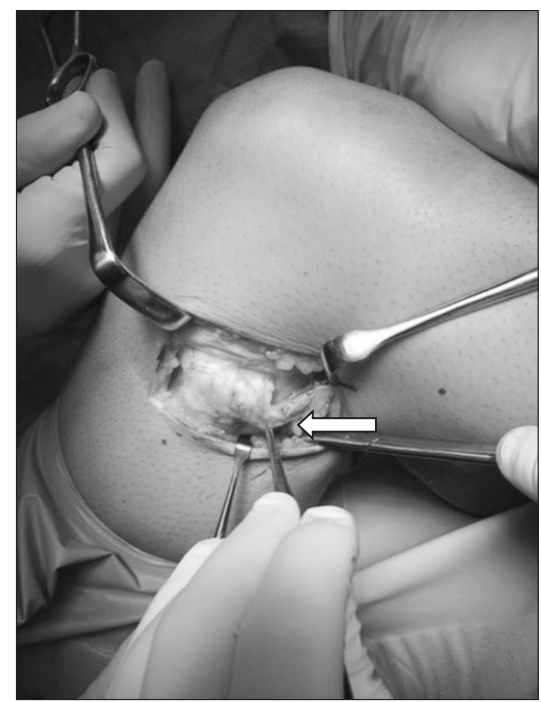

Fig. 4. Intraoperative picture of hypertrophied reflected arm of the biceps femoris tendon dissected.

was drilled through the fibular head. The detached portion of the biceps tendon was then shuttled through this tunnel using the free ends of the suture whipstitched on its terminal portion and tied to a metallic button (Fig. 6) to dock it in the socket created. The knee was brought back into extension and as the biceps tendon was quite tight coming into full extension, it was lengthened slightly using a pie crusting technique. The knee could then be easily brought into normal hyperextension. The wound was closed with monocryl (Ethicon).

Postoperatively, the knee was put in a ROM brace blocking terminal $30^{\circ}$ of extension and was mobilised non-weight bearing

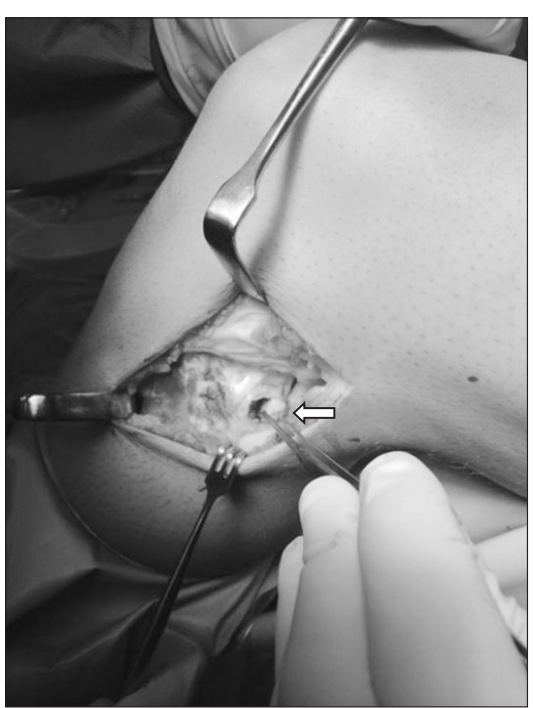

Fig. 5. Intraoperative picture of osteoplasty of the fibular head.

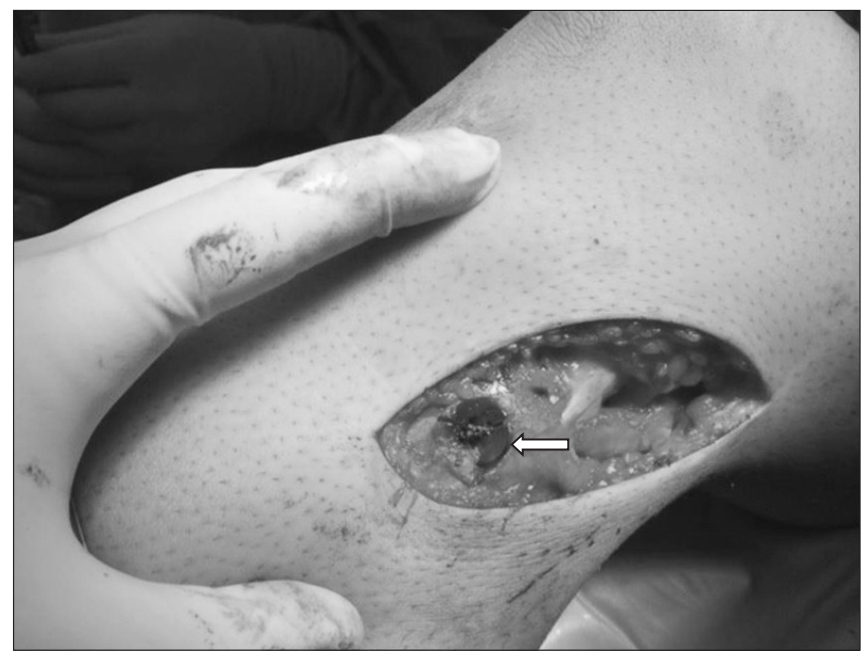

Fig. 6. Intraoperative picture of the re-attached biceps femoris tendon.

using crutches for 2 weeks. He was then rehabilitated to get full extension by prescribing active and active assisted exercises using a rolled towel under the heel with the knee in extension and asking the patient to extend the knee further. He was prevented from doing active hamstring contractions for 6 weeks: this was done by locking the knee in extension for 2 weeks while walking and releasing it at rest for passive flexion. At the 6 week mark, the ROM was normal, including slight hyperextension, and the patient was allowed to commence active hamstring contractions and gentle running. He returned to full sporting activities by 3 months after surgery and had a ROM of $0^{\circ}-130^{\circ}$. At the one-year follow-up, the patient had a visual analog scale score of 0 and no recurrence of symptoms, and he was fully functional in day to day activities as well as playing club level basketball. 


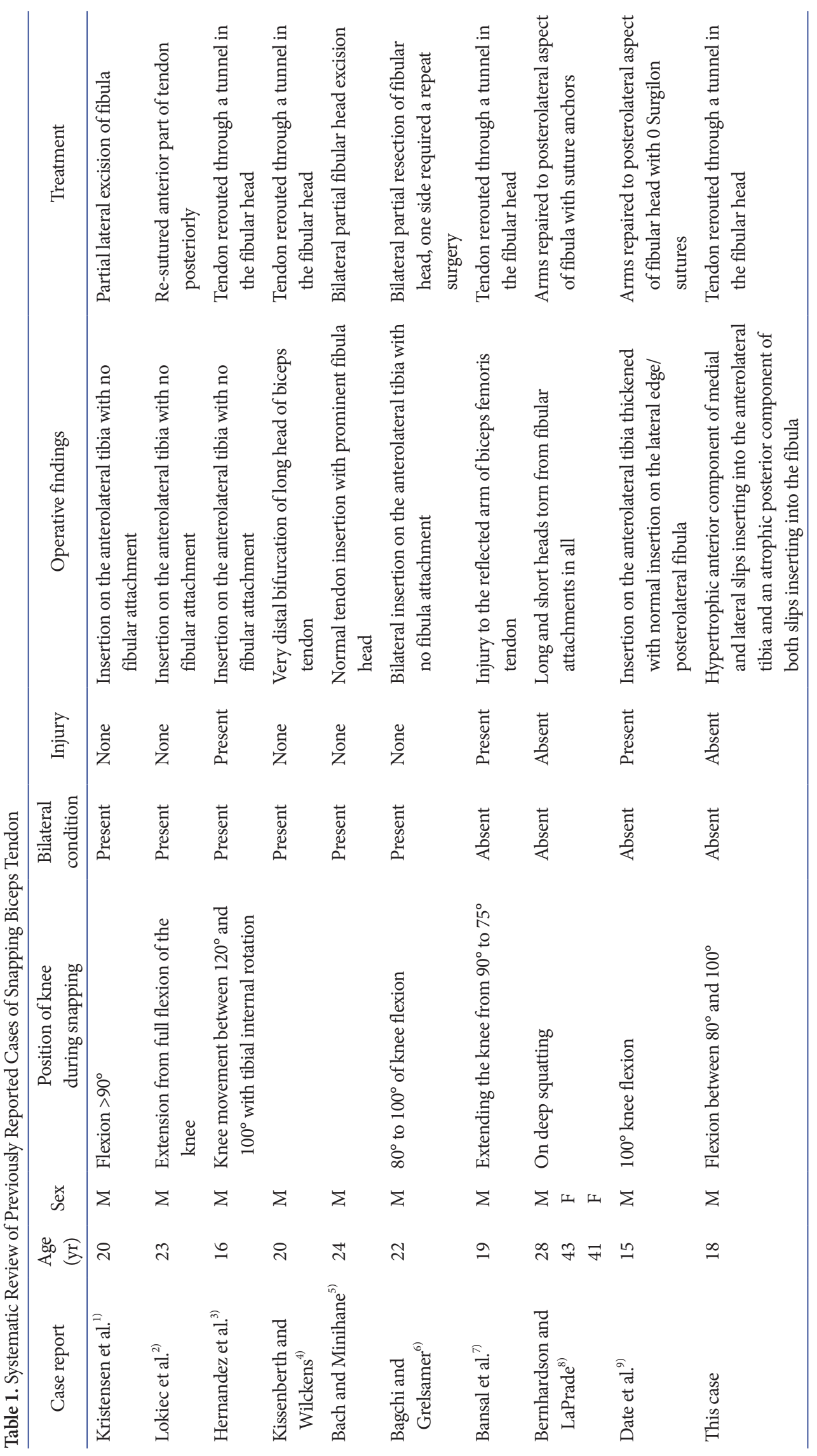




\section{Discussion}

This case describes an anomaly in the insertion of the biceps femoris tendon that has never been described in the literature. In the patient, there was a hypertrophic insertion on the tibia and an atrophic insertion on the fibula, which was successfully managed surgically.

Snapping biceps tendon is an infrequently reported condition. There have been previous case reports published ${ }^{1-9)}$, describing different anatomical variants associated with the snapping, and different approaches to its treatment. A systematic review of previously reported cases and their findings are shown in Table 1.

Date et al. ${ }^{9)}$ reported a case of snapping biceps tendon with an anomalous insertion of the anterior and reflected arms of the tendon. They were both detached from their anomalous locations and were tenodised to the periosteum on the posterolateral portion of the fibula. In a case of bilateral snapping reported by Bagchi and Grelsamer ${ }^{6}$, due to an anomalous insertion into the fascia on the anterolateral tibia rather than the posterolateral fibula, partial resection of the fibular head was performed bilaterally for treatment without reinserting the tendon to its anatomical location. An anomalous tibial attachment of the biceps tendon was also reported by Kristensen et al. ${ }^{1}$, which was treated by partial resection of the lateral part of the fibula. Hernandez et al. ${ }^{3)}$ reported a case where the phenomenon occurred due to the presence of only a tibial attachment of the biceps femoris tendon without any fibular insertion. They treated it by anatomical repositioning and tenodesis to the posterolateral fibula through a 10 $\mathrm{mm}$ drill hole and sutured to the periosteum.

These cases highlight that several different anatomical variations can result in the same symptom of "snapping bicep". Successful treatment hinges on identification of the particular individual anatomical variant and its correction in such a way that allows for normal biceps function without undue mechanical irritation of the whole complex as the knee flexes and extends. This case is unique as it describes for the first time a case of hypertrophic anterior component attaching on the anterolateral fibula and an atrophic posterior component that was attached to the fibula, which was not responsive to conservative treatment and was successfully treated surgically. Also, the anatomy of the abnormality has been reported with reference to the normal anatomy using intraoperative photographs and illustrations.

This condition is most frequently reported in younger, active males mostly in their second and third decades. The most common causative factor is an anatomical variation either in the insertion of the biceps tendon or of a prominent fibular head.
A possible reason for it to manifest around the end of the second decade of life could be the activity levels of this age group combined with specific changes that occur during fusion of the epiphyses in that area at that time. Repeated microtrauma to an existing abnormal tendon insertion may lead to hypertrophy of one or more bands of insertion or the fibular head itself. The insertion of the biceps femoris tendon in these discrete locations makes it susceptible to minor changes in the anatomy resulting in aberrations in the way the tendon glides over these surfaces during flexion and extension. Pain also accompanies the condition when inflammation occurs around the tendon due to repeated snapping.

Successful treatment was achieved in this case by recreating the attachment of the tendon that does not involve excessive friction with the adjacent bone via an osteoplasty of the fibular head and a re-routing procedure of the biceps femoris tendon.

\section{Conflict of Interest}

No potential conflict of interest relevant to this article was reported.

\section{References}

1. Kristensen G, Nielsen K, Blyme PJ. Snapping knee from biceps femoris tendon: a case report. Acta Orthop Scand. 1989;60:621.

2. Lokiec F, Velkes S, Schindler A, Pritsch M. The snapping biceps femoris syndrome. Clin Orthop Relat Res. 1992;(283): 205-6.

3. Hernandez JA, Rius M, Noonan KJ. Snapping knee from anomalous biceps femoris tendon insertion: a case report. Iowa Orthop J. 1996;16:161-3.

4. Kissenberth MJ, Wilckens JH. The snapping biceps femoris tendon. Am J Knee Surg. 2000;13:25-8.

5. Bach BR Jr, Minihane K. Subluxating biceps femoris tendon: an unusual case of lateral knee pain in a soccer athlete: a case report. Am J Sports Med. 2001;29:93-5.

6. Bagchi K, Grelsamer RP. Partial fibular head resection for bilateral snapping biceps femoris tendon. Orthopedics. 2003; 26:1147-9.

7. Bansal R, Taylor C, Pimpalnerkar AL. Snapping knee: an unusual biceps femoris tendon injury. Knee. 2005;12:458-60.

8. Bernhardson AS, LaPrade RF. Snapping biceps femoris tendon treated with an anatomic repair. Knee Surg Sports Traumatol Arthrosc. 2010;18:1110-2. 
9. Date H, Hayakawa K, Nakagawa K, Yamada H. Snapping knee due to the biceps femoris tendon treated with repositioning of the anomalous tibial insertion. Knee Surg Sports Traumatol Arthrosc. 2012;20:1581-3.
10. Terry GC, LaPrade RF. The biceps femoris muscle complex at the knee: its anatomy and injury patterns associated with acute anterolateral-anteromedial rotatory instability. Am J Sports Med. 1996;24:2-8. 